\title{
INVESTIGACIONES
}

\section{Las relaciones de otredad, alteridad e inclusión identitaria cultural de estudiantes inmigrantes en espacios escolares municipalizados en la región metropolitana}

\author{
The relationships of otherness, otherness and cultural identity inclusion \\ of immigrant students in municipal school spaces in the metropolitan region
}

\section{Luis Enrique Pincheira Muñoz ${ }^{a}$}

${ }^{a}$ Investigador asociado Centro de Estudios Latinoamericano de Educación Inclusiva CELEI- Chile. pincheira.luis@gmail.com

\begin{abstract}
RESUMEN
El artículo describe los hallazgos de una tesis doctoral dirigida a la interpretación de la integración cultural de los estudiantes inmigrantes en dos escuelas chilena, así como representaciones de docentes, directivos, docentes de aula, profesionales no docentes, padres y/o apoderados y estudiantes sobre las relaciones de Otredad, valorización de la Alteridad e Identidad Cultural. Hallazgos: Ambas escuelas las relaciones de Otredad con el estudiante inmigrante, son de carácter superficial, falta profundizar las características propias de diferenciación tales como: Psicosociales y Socioculturales. En relación con la Alteridad, ambas instituciones, no está instalada la comprensión del cambio en la alternancia recíproca de las culturas que reconoce al distinto como un interlocutor válido en un proyecto dialógico, ético y político. Finalmente, la valorización de la Identidad Cultural, en ambas instituciones prevalece un enfoque asimilador de adaptación cultural, lo cual implica una sumisión e identificación al sistema de valores de la cultura que acoge.
\end{abstract}

Palabras claves: Otredad, alteridad, identidad cultural, estudiante inmigrante, escuela municipal.

\section{ABSTRACT}

The article describes the findings of a doctoral thesis aimed at interpreting the cultural integration of immigrant students in two Chilean schools, as well as representations of teachers, managers, classroom teachers, nonteaching professionals, parents and/or guardians and students on the relations of Otherness, appreciation of Otherness and Cultural Identity. Findings: Both schools the relations of Otherness with the immigrant student are of superficial character, lack to deepen the own characteristics of differentiation such as: Psychosocial and Sociocultural. In relation to the Otherness, both institutions, there is not installed the understanding of the change in the reciprocal alternation of the cultures that recognizes the different one as a valid interlocutor in a dialogical, ethical and political project. Finally, the valuation of the Cultural Identity, in both institutions prevails an assimilating approach of cultural adaptation, which implies submission and identification to the system of values of the culture that receives.

Key words: Otherness, alterity, cultural identity, immigrant student, municipal school. 
Estudios Pedagógicos XLVII N² 2: 355-369, 2021

LAS RELACIONES DE OTREDAD, ALTERIDAD E INCLUSIÓN IDENTITARIA CULTURAL DE ESTUDIANTES

INMIGRANTES EN ESPACIOS ESCOLARES MUNICIPALIZADOS EN LA REGIÓN METROPOLITANA

\section{INTRODUCCIÓN}

El ingreso del estudiante inmigrante es un desafío para la escuela municipal en tanto que espacio de reproducción y validación de discursos, dinámicas y prácticas diversas de convivencia social escolar. En esta realidad, no pueden quedar al margen del análisis las representaciones de estudiantes de otras nacionalidades, en este caso la inmigración peruana inserta en la escuela pública chilena, puesto que en esta instancia se desarrollan y se generan aprendizajes y diálogos ligados a la representación del Otro.

La contextualización del fenómeno migratorio en las escuelas municipalizadas es relevante, debido a la escasa información y sistematización de esta.

Según, Fernández (2012):

[...]No existe estadística precisa y actualizada respecto a la situación migratoria, tampoco sobre sus principales características. Esto genera que hay escaso conocimiento sobre los focos que requieren mayor atención, como podría ser la inserción educacional, el acceso a servicios locales su integración cultural. (p. 242).

La contribución de esta investigación es dar a conocer las representaciones, de los actores invitados como primera fuente informativa, nos parece de vital importancia porque explicitan visiones del trabajo cotidiano sobre la diversidad cultural, en sus prácticas pedagógicas y, a la vez, conocer los argumentos de las implementaciones e innovaciones realizada estos años.

El primer subtema se refiere al resumido compilado de la revisión de la literatura que explicitan los fundamentos teóricos del estudio. El segundo subtema se desglosa la metodología empleada. Como tercer subtema, se desprende al análisis y discusión de los grupos participantes que procura determinar el grado de aproximación o distancia que tienen las representaciones discursivas con las categorías conceptuales teóricas. Y finalmente se presentan las conclusiones del estudio.

\section{REVISIÓN DE LA LITERATURA}

A continuación, se presenta un resumido compilado de los siguientes referentes que fueron utilizadas como categorías conceptuales del estudio.

El primer autor referente, es Tzvetan Todorov, el cual realiza un análisis interesante en relación con las relaciones de Otredad, manifiesta que no siempre se realiza un análisis profundo de dichas relaciones a diario con el Otro, más aún, todos vivimos habitados por el otro en forma de pareja, familia, comunidad, país, migrantes, en resumidas cuentas, de culturas.

Todorov, (2003) nos introduce en la historia del llamado de las preocupaciones sobre las relaciones de Otredad, el cual afirma:

[...] El descubrimiento que el yo hace del otro, implica entrar en una complejidad psicosocial compleja. Apenas lo fórmula uno en su generalidad, ve que se subdivide en categorías y en direcciones múltiples, infinitas. Uno puede descubrir a los otros en uno mismo, darse cuenta de que no somos una sustancia homogénea, y radicalmente extraña a todo lo que no es uno mismo: [...] este grupo puede a su vez, estar en el 
interior de la sociedad: las mujeres para los hombres, los ricos para los pobres, los locos para los "normales"; o puede ser exterior a ella, es decir, otra sociedad que será, según los casos, cercana o lejana; seres que todo lo acerca a nosotros en el plano cultural, moral, histórico o bien, son desconocidos, extranjeros cuya lengua y costumbres no entiendo, tan extranjeros que, en el caso límite, dudo en reconocer nuestra pertenencia común a una misma especie. (p. 13).

Por su parte, Garduño (2010) afirma:

[...] Todorov, propone demostrar lo siguiente: 1) Que la conquista de América vaticina y establece nuestra identidad presente y nuestra nueva relación con el Otro. 2) El triunfo de la Conquista se debió, primero, al "arte de la adaptación y la improvisación" de los conquistadores; segundo, a la superioridad en la comunicación de los signos, y tercero, al hecho de que en las múltiples combinaciones de la triada amor-conquistaconocimiento (sobre la Otredad) subyace la firme convicción de la superioridad europea y en forma consecutiva la asimilación de los nativos. (p. 181).

Nuestro segundo autor, es Enrique Dussel filosofo latinoamericano el cual se refiere a que la Alteridad, es una propuesta, más allá de la modernidad europea y la dependencia cultural propia de Latinoamérica, "es descubrir un camino que se va trazando en la praxis liberadora del pueblo" (p. 84). Es decir, la Alteridad se realiza en el hombre con el afán de buscar la liberación no por las vías de la violencia sino por la vía del reconocimiento, el diálogo y el consenso.

En el libro método para una filosofía de la liberación Dussel (1974) menciona:

[...] El Otro es América Latina con respecto a la totalidad europea, desde la perspectiva de la Alteridad. "la noción del otro se refiere a la existencia de un sujeto distinto de mi yo, la existencia de un yo que no soy yo». Esta constatación del otro plantea dos tipos de problemas. a. El conocimiento del Otro yo, entendido no como objeto, sino como otro sujeto. b. La comunicación intersubjetiva, entre yo y el otro. (p. 176).

Para Dussel, el objetivo principal de la Alteridad es romper con las totalidades que esclavizan al hombre e impiden reconocer al Otro como persona, considerando la pedagogía como una de las muchas salidas viables. Se hace necesario aclarar que cuando se habla del Otro puede ser comprendido no solo como un ser individual sino también como un pueblo, una cultura, una nación, un migrante, un continente e incluso a toda la humanidad.

En síntesis, la Alteridad que plantea Dussel, dota a cada ser humano de la valoración ante la realidad de un Otro, elemento esencial y fundamental, para construir una sociedad comunitaria que sea la contraparte de las exclusiones, guerras y violencias que vemos en el mundo, fruto de las maltrechas relaciones entre los seres humanos producto de una Alteridad alterada y dañada.

Y, por último, nuestro tercer autor, Colombres, que cita a Bonfil (1984), sostiene que la Identidad cultural:

[...] El problema consiste en definir una relación significativa entre sociedad y cultura para entender mejor los procesos culturales que ocurren, cuando dos grupos con 
Estudios Pedagógicos XLVII Nº 2: 355-369, 2021

LAS RELACIONES DE OTREDAD, ALTERIDAD E INCLUSIÓN IDENTITARIA CULTURAL DE ESTUDIANTES

INMIGRANTES EN ESPACIOS ESCOLARES MUNICIPALIZADOS EN LA REGIÓN METROPOLITANA

culturas diferentes e identidades contrastivas están vinculados por relaciones asimétricas (dominación/subordinación). Menciona como elementos culturales todos los recursos de una cultura que son necesarios para formular y realizar un proyecto social distinguiendo las siguientes clases de elementos a) Materiales, los naturales transformados por el hombre, b) de Organización, que se refieren a las relaciones sociales sistematizadas, c) de conocimiento, es decir, experiencias asimiladas y sistematizadas y las capacidades de crear, d) simbólicos, códigos de comunicación y representación, signos y símbolos; y, finalmente, e) emotivos tales como los sentimientos, valores y la subjetividad como recurso. (p. 1).

Continúa diciendo que todo proyecto social, requiere de estos elementos de identidad cultural para hacer posible el proyecto, la relación que se establece entre quién (grupo social) decide y sobre qué elementos culturales (p. 2).

Cuando una relación entre grupos con culturas diferentes es posible distinguir en la cultura del grupo subalterno la presencia de elementos culturales en cada uno de los cuatros ámbitos de la cultura dominante: autónoma, apropiada, enajenada e impuesta.

Tal como lo plantea Colombres (1984) en este caso, el estudiante inmigrante inserto en la escuela pública en Chile, la cultura es una sola contradictoria, híbrida si se quiere, pero al analizar en términos de control cultural, aparece su composición en los cuatros ámbitos.

Como el problema está definido en términos de relaciones entre grupos sociales la dialéctica del control cultural no se establece entre "lo mío" y "lo tuyo" sino entre lo "nuestro" y "de los otros" lo propio y ajeno tiene una connotación social y no individual.

Es decir, en el estudio realizado en estas dos escuelas municipales, hay una cultura impuesta, ni las decisiones, ni los elementos culturales puesto en juego son del grupo social, en este caso, los estudiantes inmigrantes, sin embargo, entran a formar parte de la cultura total del propio grupo tales como hábitos de consumo, modelos de vida, valores, aspiraciones y el propio sistema educativo; en este caso, se incorporan elementos culturales que permanecen ajenos porque su control no es de la comunidad considerada. Según Colombres, que cita a Bonfil "la clase subalterna, en este caso, el estudiante inmigrante sufre exclusión de las decisiones sobre los elementos culturales que desarrolla la institución escolar con la administración del curriculum nacional en beneficio de la cultura dominante" (p. 5).

\section{METODOLOGÍA}

La investigación siguió un paradigma cualitativo con prevalencia de herramientas descriptivas e interpretativas, es decir, se realiza un análisis desde la cultura de los actores a investigar, tal como Rodríguez, Flores y Jiménez (1996) manifiestan: "hacer coherente lo que de otra forma aparece un conjunto de hechos desconectados" (p. 81). El método utilizado, fue un estudio de caso de carácter colectivo o múltiple para profundizar las "proximidades", "lejanías", "similitudes" y "discrepancias" del fenómeno de interés del investigador. Rodríguez et al. (1996) afirman: "el estudio de caso implica un proceso de indagación que se caracteriza por el examen detallado, comprensivo, sistemático y en profundidad del caso objeto de interés" (p. 93). 


\subsection{CONTEXTO}

El contexto se sitúa en dos escuelas públicas (X, Y) en las comunas de Santiago Centro e Independencia de la región Metropolitana, cuyo porcentaje de estudiantes inmigrantes prevalece sobre el $50 \%$ en la matrícula como alumno/a regular.

En relación con los participantes, se consideró a todos los grupos representativos de la comunidad educativa: Docentes Directivos, Docentes de aula, Profesionales no docentes, padre, madre y/o apoderados/as y estudiantes sin discriminar ni por género ni por edad. La muestra correspondió a 15 personas por cada escuela.

Como criterio de participación, se consideró la permanecía laboral más de cinco años en la escuela y se conformaron los siguientes grupos: Docentes Directivos, cuya responsabilidad es de carácter de Gestión y Técnico Administrativo de la escuela tales como: Director, Subdirector/a, Jefe de Unidad Técnico-Pedagógica e Inspector General. El grupo docentes, se conformó por el Profesor/a Jefe/a del curso Séptimo y Octavo y los docentes con mayor carga horaria en los subsectores de Lenguaje y Comunicación, Matemáticas, Comprensión del Medio y Comprensión de la Sociedad. En cambio, el grupo de Profesionales no docentes se invitó a participar al Psicólogo/a, y el asistente de la educación.

Por otro lado, el grupo de Padre, madre y/o apoderados/as fueron personas con dilatada colaboración y participación en el curso de séptimo y octavo. Y finalmente, el grupo de estudiantes se realizó un llamado abierto en los cursos de séptimo y octavo básico, que quisieran compartir sus experiencias y colaborar con la investigación.

\subsection{INSTRUMENTO}

Se utilizó la entrevista semiestructurada como técnica que implica un proceso de interacción entre el entrevistador y entrevistado donde se despliega una historia personal, que forma parte de una historia colectiva. Ríos (2013) afirma:

[...]Es una técnica de tipo directa basada en la interacción dinámica entre dos personas: entrevistador y entrevistado, cuyo control se encuentra en el primero de estos. Posee un carácter holístico en el cual el objeto de investigación es la vida, las experiencias, las ideas, los valores y la estructura simbólica del entrevistado. (p. 113).

\subsection{PROCEDIMIENTO}

Fue análisis del discurso, como herramienta, visualizando el entorno en que se movilizan los individuos investigados, donde el lenguaje juega un rol central, un puente para establecer vínculos y relaciones sociales. Este análisis no solo parte de la oralidad de lo representado por los actores participantes, se amplía la mirada de los signos transcrito, además se considera el contexto social que está inmerso los participantes.

Santander afirma:

[...] El Análisis del Discurso parte de la base que la lengua (escrita y oral) no es el medio exclusivo de representación y comunicación, de lo contrario, el AD no se distinguiría de la Lingüística Textual... semiótica (o semiótica a secas) amplía su 
Estudios Pedagógicos XLVII Nº 2: 355-369, 2021

LAS RELACIONES DE OTREDAD, ALTERIDAD E INCLUSIÓN IDENTITARIA CULTURAL DE ESTUDIANTES

INMIGRANTES EN ESPACIOS ESCOLARES MUNICIPALIZADOS EN LA REGIÓN METROPOLITANA

mirada tanto a signos de otra naturaleza como a la relación de esos signos con los contextos sociales, extralingüísticos. (p. 214).

\section{ANALISIS Y DISCUSIÓN}

En este apartado, se exponen los discursos y las prácticas de los diversos grupos de actores de la escuela X y su interpretación comprensiva con los referentes teóricos que se utilizaron como marco referencia.

En cuanto a relaciones de otredad, el grupo de directivos considera que se ha logrado instalar un protocolo de acogida y acciones formativas con temas asociados a: respeto, aceptación y tolerancia entre estudiantes; medidas implementadas, que tienen su fundamento en las relaciones de "Otredad", para ser respetada/o desde su originalidad en el ámbito de las relaciones humanas.

Desde la propuesta de Todorov, este grupo reconoce sus rasgos externos, que los hace extranjeros con respecto a su par chileno que tienen los mismos derechos y oportunidades al ser recibidos en la escuela.

Podemos, afirmar que el discurso presenta un interés de reconocimiento desde la exterioridad con una mirada humanizadora en el derecho a la educación para todo estudiante. Aún no está instalada la comprensión de una etapa de profundización hacia una valoración de la diferenciación de las características propias del estudiante inmigrante que aporta en sus relaciones de "Otredad" para implementar una verdadera "inclusión escolar" que plantea la aceptación a la diversidad cultural.

Por su parte, el grupo de los docentes expresa que las relaciones de "Otredad" es un proceso que no significa un simple estar junto, sino es un proceso de profundización interactivo que exige contacto, valoración y relaciones de intercambio positivas entre personas; en este caso, alumnos en el aula en un clima de diversidad cultural. Aprender a vivir juntos en el contexto escolar significa tolerar la existencia de un otro ser humano culturalmente distinto, esto significa un tiempo prolongado para respetarlo, conocerlo y valorarlo en un clima de cercanía cotidiana entre personas con un bagaje en su cultural distinto.

En general, los docentes afirman que el colegio es pequeño en su infraestructura física, lo cual hace que la multiplicidad de interacciones en lo cotidiano a nivel de adultos y niños y jóvenes y todos los actores, sea de carácter familiar en la cultura escolar, lo cual permite una aceptación y reconocimiento.

El grupo considera la complejidad de la relación de "Otredad" en el ámbito escolar, comparte un acercamiento parcial al planteamiento de Todorov, lo difícil de identificar las características propias de diferenciación en los seres humanos, dado que se necesita tiempo y conocimiento.

A modo de concluir, el grupo de docentes, en sus argumentos manifiesta que hay un reconocimiento parcial en las relaciones de "Otredad" del estudiante inmigrante, ya que al mismo tiempo agregan que, por razones programáticas oficiales, la inclusión del "Otro" que trae un distinto proceso formativo en su contenido escolar, aún no ha sido posible integrar. Es decir, reconoce la amplitud y profundidad de las relaciones de "Otredad"

Se refiere al estudiante inmigrante. 
pero a la vez reconocen la dificultad de asimilación a la condición académica de sus pares chilenos.

En lo que respecta al grupo de profesionales no docentes, declara que las relaciones con un otro diferente, implica un trabajo colaborativo respetando su diversidad de intereses en las múltiples actividades que se comparte. De alguna forma hay un acercamiento a lo que plantea Todorov (2003) el cual manifiesta: "puede ser un grupo social concreto al que nosotros no pertenecemos que puede estar al interior de la sociedad: las mujeres para los hombres o puede ser exterior a ella, es decir otra sociedad que será en algunos casos cercano o lejano" (p. 13). Pero, el reconocimiento de la diversidad del otro no implica la comprensión real de las relaciones de "Otredad".

El grupo de padres y/o apoderados, de acuerdo con lo que postula Todorov, en sus discursos, considera que hay un reconocimiento superficial en las relaciones de "Otredad" del estudiante inmigrante, que se debe incrementar en todas las actividades socioculturales para identificar las características propias de diferenciación de cada cultura para ser verdaderos protagonistas de encuentros con el "Otro" y no meros acompañantes en los pocos eventos que se realizan en la escuela en el año escolar.

Al concluir, este grupo tiene una visión crítica sobre el reconocimiento de las relaciones de "Otredad" del estudiante inmigrante, considera que aún no se ha permitido desarrollar espacios para dar a conocer todas las culturas que cohabitan. Tal como, Todorov afirma: "el tema de la Otredad es inmenso, apenas lo fórmula uno en su generalidad, ve que se subdivide en categorías y en direcciones múltiples infinitas" (p. 13).

En cambio, el grupo de estudiantes: manifiesta que las relaciones son múltiples y variadas en lo cotidiano que la escuela, solo hay un conocimiento muy superficial de las distintas culturas, y que aquellos que dirigen y los docentes deben profundizar lo realizado hasta el momento.

Para concluir, los diferentes grupos de la escuela $\mathrm{X}$ destacan sobre las relaciones de "Otredad" del estudiante inmigrante, se puede inferir que prevalece lo que plantea Todorov, en los discursos está implícito un reconocimiento superficial, se prioriza una visión anecdótica de la Otredad, con una conceptualización errada de lo que significa las relaciones de "Otredad" la cual se confunde conceptualmente con el término de un reconocimiento de otro distinto; en este sentido, la comunidad educativa tiene el desafío de lograr esclarecer conceptualmente qué significa las relaciones de "Otredad" cultural, tal como lo plantea Todorov, "hablar del descubrimiento que el yo hace del otro es un tema inmenso, porque no se puede hablar de todo a la vez sobre el tema" (p. 13).

En relación con la Alteridad, el grupo de directivos, reconocen el impacto en la comunidad educativa de la cultura peruana de su cosmovisión "andina" o "afroperuana" se podría decir un acercamiento al planteamiento de Dussel sobre la Alteridad, la valorización de los peruanos, en desmedro de los otros grupos culturales minoritarios, subordinados a la simetría cultural chilena-peruana.

Por su parte, el grupo de docentes, en la visión de Dussel, en sus argumentos hay un intento de reconocer la Alteridad del estudiante inmigrante como un otro intersubjetivo que enriquece con su diversidad; se transita inicialmente en una etapa de experimentación, no es menos cierto recalcar que esta comunidad escolar lleva más de una década con estudiantes inmigrantes en el aula, cuyas experiencias educativas aisladas son la punta del "iceberg".

Por su parte, el grupo de profesionales no docentes manifiesta al igual que los otros grupos, un reconocimiento del impacto de la cultura peruana en la comunidad escolar, ha 
sido un reto importante de socialización en un clima de diversidad cultural, tal impacto de "peruanización" ha logrado que en las personas chilenas y de otros países, internalicen esos elementos culturales en la cotidianidad, como, por ejemplo: uso de nuevas palabras, celebraciones gastronómicas, que ha enriquecido a estudiantes y adultos.

Podríamos decir que este grupo, en el pensamiento de Dussel sobre la Alteridad, logran dar importancia a una valorización de un Otro yo, entendido no como objeto, sino como otro sujeto, dicho reconocimiento es una mirada "estética e instrumental" un "maquillaje" de vivencias concretas realizadas por la comunidad peruana, quedando al olvido otros sujetos de las culturales minoritarias que conviven.

En lo que atañe al grupo de padres y/o apoderados, la opinión generalizada (chilenos e inmigrantes) estos últimos años hay un reconocimiento en conocer sus elementos culturales de las nacionalidades pertinentes, consideran la importancia de ser valorado de un modo visible. Para este grupo, se debe desarrollar un proceso de mayor profundización dialógica entre todos los actores de la comunidad escolar, que incluya en una participación colegiada, para lograr una verdadera valorización de la Alteridad de los diferentes estamentos.

En cambio, el grupo de estudiantes manifiestan que hay un reconocimiento superficial de la Alteridad del otro, cuya base son esas relaciones cotidianas, a través de la comunicación instrumental entre sujetos en la cotidianidad.

Por consiguiente, los diferentes grupos de la Escuela $\mathrm{X}$ en sus representaciones discursivas sobre la Alteridad del estudiante inmigrante hay visiones diferenciadas y superficiales sobre la valoración de un otro cultural que me puede enriquecer. Frente a estas visiones diferenciadas hay un consenso general de valorizar la Alteridad del estudiante peruano como un sujeto valioso y su impacto cultural en la comunidad educativa, pero se invisibilizar en desmedro las otras Alteridades no peruanas.

En relación a la Identidad cultural, el grupo de directivos, en sus representaciones discursivas, prevalece una aproximación a dos variables de identidad cultural que nos señala nuestro autor elegido Bonfil: En primer lugar hay un reconocimiento de la materialidad cultural del estudiante inmigrante, que se manifiesta, a través de los diversos eventos públicos y cotidianos en que participa junto a sus familias y, en segundo lugar, hay cierta valoración de los elementos emocionales o subjetivos del estudiante inmigrante con respecto a su par chileno; en este grupo aún no está la comprensión de todo los elementos que configuran la identidad cultural, tales como: los conocimientos, la organización/jerarquización cultural y los elementos simbólicos importantes que portan los estudiantes inmigrantes.

En cambio, el grupo de docentes considera que la valorización de la identidad cultural del estudiante inmigrante se desarrolla en la práctica, a través de los eventos masivos internos o externos socioculturales. En este sentido, sus argumentos como señala Bonfil Batalla se remiten a la variable del reconocimiento de la identidad material que se traduce en una folklorización estética.

Por su parte, el grupo de los profesionales no docentes considera que los eventos escolares internos realizados, han logrado conocer su identidad cultural y aprender sus bailes, comidas, modismos y palabras que se han incorporado a la comunidad, y que estos actos son un reconocimiento explícito hacia el estudiante inmigrante. En síntesis, al igual que los grupos anteriores, está instalado el reconocimiento de la materialidad cultural como única variable.

Aunque, el grupo de padres y/o apoderados, consideran importante la variable que se relaciona con la "materialidad de la cultura" es primordial dar a conocer a la comunidad 
todas las identidades culturales en los eventos que se realizan en el año; ellos sienten que esos eventos son un comienzo de valoración e integración de su cultura originaria; sin embargo, todavía falta una mayor profundización de sus aportes culturales a la cultura hegemónica chilena.

Conviene señalar que el grupo de estudiantes considera importante los eventos socioculturales que se realizan, ya que están referidos a la variable de la "materialidad de la cultura" estos eventos de alguna forma es un comenzar de conocer las identidades culturales de su formación escolar.

Para finalizar, con la escuela X existe un consenso transversal de todos los grupos de valorizar la identidad cultural del estudiante inmigrante, a través de la "materialidad de la cultura" hay cierto conformismo de lo realizado, a pesar de los años con su experiencia educativa, no se observa un mayor interés en profundizar conocimientos sobre las diversas identidades culturales, que permita dar un vuelco en su proyecto educativo como institución escolar.

En el siguiente apartado, se exponen los discursos y las prácticas de los diversos grupos de actores de la escuela Y, y su interpretación comprensiva con los referentes teóricos que se utilizaron como marco referencial.

Dicho esto, en las relaciones de "Otredad", el grupo de directivos afirma que ha realizado un largo proceso de sistematización para la acogida, aceptación del estudiante inmigrante como otro diferente culturalmente, pero ese reconocimiento solo hay una comprensión desde la exterioridad de ese otro, aún falta como lo plantea Todorov, ahondar en las características íntimas y psicosociales que tiene ese otro.

A modo de síntesis, este grupo, desde sus representaciones han logrado desarrollar relaciones formales y superficiales con el estudiante inmigrante; pero, establecer con esos estudiantes relaciones de otredad que permitan incluirlos con sus características íntimas y culturales propias al proceso formativo, todavía no se reconocen. Ese reconocer y acoger al otro distinto culturalmente, se limita a los esfuerzos de aceptarlos como personas que tienen el derecho a recibir una educación, similar a cualquier otro estudiante; pero falta la iniciación de un proceso que consigne una valoración de diferenciación de las características propias del estudiante inmigrante en su "Otredad". Burocráticamente, se da cumplimiento a la ley de incluirlos escolarmente, pero incorporarlos como sujetos que tienen un aporte cultural a realizar para mejorar la calidad de la educación, todavía no está en el horizonte "inclusivo" de este grupo de Directivos. Tal inclusión "verdadera" significaría aceptar y valorar la diversidad cultural de los estudiantes en el contexto educativo.

Por su parte, el grupo de los docentes, consideran que el reconociendo de las relaciones de "Otredad" se manifiestan en la convivencia cotidiana, donde el estudiante inmigrante es un actor participante en actividades formales e informales extracurriculares.

El grupo considera complejo el proceso de la relación de "Otredad", para reconocer al estudiante inmigrante en sus características psicosociales y culturales diversas e íntimas, debido a que no se tiene la capacitación profesional para entender y discriminar positivamente sobre esos aportes diferenciados. En ese discurso auto justificativo, reconoce la importancia de configurar una escuela que sepa trabajar con la realidad diversa y particular de los estudiantes, para trabajar a nivel de aula y en las actividades extraprogramáticas, consideran adolecer de un conocimiento teórico pedagógico en el conocimiento, el cual hace enredarse en su función docente. Concuerdan, con el planteamiento de Todorov, lo difícil identificar las características propias de diferenciación de la "Otredad", se necesita tiempo y conocimiento. 
Estudios Pedagógicos XLVII N²: 355-369, 2021

LAS RELACIONES DE OTREDAD, ALTERIDAD E INCLUSIÓN IDENTITARIA CULTURAL DE ESTUDIANTES INMIGRANTES EN ESPACIOS ESCOLARES MUNICIPALIZADOS EN LA REGIÓN METROPOLITANA

Al contrario, el grupo de profesionales no docentes, reconocen a los grupos respetando su diversidad en las relaciones de "Otredad" con el estudiante inmigrante, el cual ha sido su participación en los eventos masivos dando a conocer su distinta cultura, igual que el grupo de docentes concuerdan con Todorov en la "necesidad de establecer relaciones de "Otredad", más profunda para conocerse en un plano cultural, moral, histórico" (p. 13).

Por su parte, el grupo de padres y/o apoderados, manifiestan vías de participación, con cierta crítica, de profundización en el reconocimiento de las relaciones de "Otredad" del estudiante inmigrante; generando espacios institucionales que permita organizar actividades socioculturales en forma sistemática, dialógica y horizontal, que permita un trabajo compartido.

Asimismo, el grupo estudiantes reconoce que se han desarrollado jornadas de capacitación en los consejos de cursos, cuyos temas han sido la aceptación y el respeto por cualquier persona adulta, joven o niño/a cuyo principal interés son las buenas relaciones en las actividades formales e informales al interior del colegio. Consideran que las relaciones de "Otredad" con el estudiante inmigrante son superficiales, dado que no todos se conocen en profundidad, existe solo un compañerismo cotidiano a través de los intereses, motivaciones y afinidades el cual cada estudiante elige a un par o grupo que opina o ve el mundo de esa manera. El grupo concuerda con Todorov (2003), "que este problema del otro exterior y lejano, no se puede hablar de todo a la vez se necesita tiempo" (p. 13).

A modo de sintetizar las representaciones discursivas de todos los grupos de la Escuela Y sobre las relaciones de "Otredad" con el estudiante inmigrante, podemos remarcar que, en la mayoría de los comentarios, hay un reconocimiento superficial de dichas relaciones con el Otro en su identidad extranjera, se acepta su diversidad; sin embargo, de inclusión activa de esa diversidad al proceso formativo, solo se prioriza desde una visión parcial del otro exterior y lejano.

En relación con la Alteridad, el grupo de directivos considera el aporte del estudiante inmigrante a la cultura social de la escuela y distingue principalmente al estudiante peruano como la incorporación de elementos comunicativos, tales como: el uso de nuevas palabras o modismo en el quehacer cotidiano, sus canciones, bailes y el ámbito gastronómico. Este grupo expresa que la cultura peruana, ha logrado impactar a la comunidad escolar de alguna forma en la cotidianidad.

En forma superficial concuerdan con Dussel sobre la Alteridad la valoración con un interlocutor diverso, en este caso el estudiante peruano en la comprensión intersubjetiva que permite, la irrupción del otro como una posibilidad de cambio y de construcción de un proyecto educativo distinto.

Este grupo, destaca los eventos culturales realizados a través de los años, cuya participación mayoritariamente son de la comunidad peruana, concuerdan con en parte con Dussel (1974) que afirma: "La Alteridad, se refiere a la existencia de un sujeto distinto de mi yo, la existencia de un yo que no soy yo" (p. 176). Pero incluso en ese acto dejan fuera a otros estudiantes inmigrantes, invisibilizados en sus Alteridades culturales minoritarias.

Como síntesis, el grupo directivos, aún no está la comprensión de valorar todos los aportes al cambio educativo y cultural del curriculum y de la identidad formativa de la escuela.

En cambio, el grupo de los docentes consideran valioso el aporte del estudiante inmigrante peruano, por su entrega en dar a conocer sus tradiciones y costumbres dicha valorización, trasciende los muros de la escuela, que incluye a los vecinos del sector Barrio Yungay, los cuales la reconocen, como la escuela de los estudiantes peruanos. Los docentes, 
consideran valiosa la Alteridad peruana, entendida como lo plantea Dussel el conocimiento del Otro yo, entendido no como objeto, sino como otro sujeto diferente al mí (aquí nos permitimos identificar el "mí" como lo chileno).

De acuerdo con el discurso del grupo de docentes, es insuficiente de hablar de una alteridad constructiva del cambio curricular/educativo que tal posibilidad sería viable, si cambiara el carácter de uniformidad cultural que tiene la Educación y el Curriculum Oficial Chileno.

Ciertamente, en sus argumentos hay intento de acciones aisladas de valorar la Alteridad del estudiante inmigrante, pero la configuración es una escuela tradicional cuyo proyecto normalizador se sostiene con la aplicación del curriculum de la cultura oficial.

En cambio, el grupo de profesionales no docentes concuerda al igual que los grupos mencionados anteriormente, hay una valoración de la Alteridad del estudiante inmigrante peruano, a través de su participación en los eventos y celebraciones masivas. Sin embargo, esta alteridad no significa que concuerden con el pensamiento de Dussel, más bien hay una caricaturización de la intersubjetividad de valorar los rasgos externos de ese otro.

Es más, el grupo de padres y/o apoderados, chilenos e inmigrantes, consideran que de alguna forma se valoriza a todo estudiante inmigrante que se integra al colegio, pero que en la práctica la comunidad peruana en forma prioritaria ha tenido las oportunidades de dar a conocer su cultura, quedando en desmedro las otras presencias culturales de estudiantes no peruanos. En sus fundamentos críticos, todavía ni los directivos, docentes y profesionales no docentes, no está la valoración del estudiante inmigrante como un eventual potencial transformador de la identidad educativa de la escuela, como Cabaluz (2015) afirma: "la Alteridad permite reconocer que en él/ella, el otro/a reside un potencial transformador" (p. 72).

En cambio, el grupo de estudiantes, en sus discursos consideran que existe múltiples opiniones de valoración con el estudiante inmigrante a nivel de pares, que se relaciona con la amistad que se pueda generar más allá de la escuela; y un segundo aspecto es la participación en conjunto en los eventos socioculturales que se realizan, conociendo su cultura del país del cual proviene.

Se puede afirmar que la alteridad dusserliana no está instalada en el discurso de los estudiantes, pero perciben que aquellas relaciones de amistad y colaboración que se dan en los espacios extraescolares y extracurriculares podrían también darse en el proceso formativo que se les destina. Sus fundamentos se aprecian en que reconocen que las interacciones variadas de convivencia y amistad al interior del colegio y fuera del colegio, se podrían también desarrollar en los espacios de formación.

Y esto es justamente lo que plantea Dussel, que la "comunicación intersubjetiva, entre yo y el otro" es la herramienta que permite dialogar, descubrirnos y reconocernos la valía que tiene cada persona cuando interactuamos con quienes nos rodean a diario en nuestras vidas.

Para finalizar, los diferentes grupos sobre la Alteridad del estudiante inmigrante, se puede inferir que hay visiones diferenciadas, estas visiones sobre la Alteridad, en primer lugar, hay un consenso en el discurso de todos los grupos del reconocimiento del estudiante inmigrante, como un sujeto valioso con su llegada a la comunidad escolar, el consenso concuerda, con lo que plantea Dussel sobre la Alteridad, "el conocimiento del Otro yo, entendido no como objeto, sino como otro sujeto".

El desafío actual de esta comunidad educativa es el tránsito a un paradigma que se orienta y se hace vida en la educación intercultural. En este sentido, la alteridad tendría el 
valor de iniciar un proceso liberador a cualquier relación de dominación o subordinación cultural hegemónica de los estudiantes inmigrantes.

Ahora bien, en relación con la Identidad cultural, el grupo directivo, reconocen en sus discursos que existen insuficiencias en el desarrollo de las manifestaciones de identidad cultural, y que este último periodo desde el 2015 se ha sistematizado acciones, tales como: cantar el himno nacional peruano el día lunes y celebrar la Independencia del Perú en el mes de julio, debido a que la gran mayoría de los/las estudiantes son de nacionalidad peruana.

Concuerdan, con dos elementos que plantea Colombres citado por Bonfil sobre la identidad cultural: el primer elemento es el reconocimiento de los aspectos de la "materialidad cultural" del estudiante inmigrante que se manifiestan a través de los eventos públicos cotidianos al interior del colegio en que participan con sus familias; y el segundo elemento. es la valorización de los aspectos "emocionales o subjetivos" aceptándolo en su diversidad con respecto a sus pares chilenos; el grupo plantea en sus fundamentos que este segundo aspecto es la puesta en marcha de la política educativa de valorar la diversidad de sus estudiantes, pero sin integrar al currículo escolar los conocimientos, la organización/ jerarquización cultural y los elementos simbólicos importantes para desarrollar la identidad cultural en plenitud.

Entonces, podríamos decir, a modo de síntesis, que en sus relatos discursivos hay un planteamiento retórico sobre la identidad cultural del estudiante inmigrante que, curiosamente, poseen como escuela: este discurso retórico presenta una mirada sesgada de las culturas, ya que no reconocen los aportes educativos-culturales que debieran tener todos/as los/las estudiantes que asisten a esta escuela. La invisibilización en la comunidad escolar de las identidades culturales inmigrantes minoritarias requiere una urgente revisión de aquellos que dirigen la institución escolar.

Por su parte, el grupo de los docentes, consideran que la identidad cultural del estudiante inmigrante se ha desarrollado en la práctica a través de los eventos socioculturales realizados. En este sentido, sus argumentos se remiten a la afirmación de los elementos sobre la "materialidad cultural" (bailes, danzas comidas etc.) y sus elementos simbólicos, tales como: (tradiciones, costumbre, lengua etc.), de la comunidad de estudiantes peruanos que es mayoritariamente.

En cambio, el grupo profesionales no docentes considera que los eventos socioculturales realizados, han sido un gran logro para conocer las identidades de las culturas inmigrantes, tales como: aprender sus bailes, degustación de comidas, palabras nuevas que se han incorporado en la comunidad.

Ellos consideran un logro del proceso realizado por varios años, asimilado en la cotidianidad de la comunidad escolar. Podríamos resumir, que este grupo, hace alusión a un reconocimiento de la identidad cultural del estudiante inmigrante peruano, pero queda claro la evidencia de no difusión y reconocimiento de otras identidades culturales de estudiantes inmigrantes no peruanos, el cual produce una asimetría en el ámbito cultural en esta institución educativa.

Por su parte, el, grupo de padre y/o apoderados, manifiestan que los eventos son llamativos y alegres en su expresión comunitaria, pero carecen de una profundización de conocer verdaderamente la identidad cultural de las diversas culturas que conviven. Esta situación se explicaría, por la predominancia de la cultura oficial hegemónica, lo que transforma las acciones promocionales artísticas y folclóricas, en un momento lúdico 
que dura en la medida en que se desarrolla. Para ellos falta un desarrollo de eventos socioculturales más frecuentes que permita dialogar y compartir todas las culturas en la comunidad escolar chilena. Estos eventos se pueden interpretar como un derecho que valida la participación de todas las personas, bajo un reconocimiento de un "Otro" diferente cultural, bajo la retórica de aquellos que dirigen con un discurso oficial "tenemos que hacer participar a todos nuestros estudiantes".

En cambio, el grupo de estudiantes solo resaltan en sus discursos el desarrollo de los eventos y actividades socioculturales que se relacionan con los aspectos materiales tales como: (bailes, danzas, comidas etc.) que nos plantea Bonfil Batalla.

Para finalizar el análisis de los diferentes grupos de la Escuela Y, en ninguno de los grupos hay una comprensión profunda de lo que implica la identidad cultural del estudiante inmigrante en el funcionamiento formativo integral. Por el contrario, sus discursos reconocen ciertas variables que suponen la identidad cultural de los/las estudiantes inmigrantes, particularmente los rasgos visibles y materiales de esa identidad, pero no hay una comprensión y una valoración de los rasgos identitarios culturales del estudiante inmigrante, tales como: el tipo de organización y de relaciones sociales que tienen en su cultura originaria, los mecanismos cognoscitivos particulares, los conocimientos diferenciales que portan en comparación con su par chileno, los componentes simbólicos de los países de origen o procedencia y, finalmente, los aspectos emocionales y subjetividades que se expresan en los sentimientos del estudiante inmigrante.

\section{CONCLUSIONES}

Estas se derivan del carácter institucional del manejo conceptual y la comprensión de las categorías teóricas sobre relaciones de Otredad, Alteridad e Identidad Cultural de este estudio.

Una primera conclusión es que en ambas comunidades educativas hay una visión errada con respecto a las relaciones de "Otredad" del estudiante inmigrante. Se acepta e incluye como un estudiante regular, se reconoce su diferencia cultural, pero no se acepta como un estudiante con sus propios ritmos de aprendizaje que ha sido formado en otros contextos socioculturales. Por lo tanto, no está presente un proceso comprensivo que reconozca y profundice las diferenciaciones socioculturales y psicosociales de la "Otredad" que tiene cada estudiante y que lo distingue como un aporte cultural distinto en la convivencia escolar cotidiana.

Una segunda conclusión es que en ambas instituciones educativas el grupo padre, madre y/o apoderados, critican la falta de voluntad institucional para propiciar acciones dialógicas y participativas de carácter horizontal, esto, a juicio de ellos, haría fluir con mayor sinceridad las confianzas y el reconocimiento de las relaciones de "Otredad" de aquellos grupos de adultos que desean ser un aporte cultural beneficioso donde estudian sus hijos/as.

Una tercera conclusión es la incomprensión conceptual de la "alteridad". Todos los actores de ambas instituciones la confunden como expresión sinónima de la alternatividad, es decir el reemplazo de la cultura chilena por la cultura que domine en el mundo inmigrante y entonces se colocan a la defensiva, afirmando el derecho de nuestro país de chilenizar a todos los extranjeros que sean admitidos en las escuelas públicas. 
Estudios Pedagógicos XLVII N² 2: 355-369, 2021

LAS RELACIONES DE OTREDAD, ALTERIDAD E INCLUSIÓN IDENTITARIA CULTURAL DE ESTUDIANTES INMIGRANTES EN ESPACIOS ESCOLARES MUNICIPALIZADOS EN LA REGIÓN METROPOLITANA

Una cuarta conclusión es que ambas instituciones educativas deben avanzar hacia una etapa que transite a la valorización del estudiante inmigrante, se trata de ahondar y caminar hacia la Alteridad, como fundamento de una Educación Intercultural.

A nuestro entender, la Alteridad, como fundamento de la Educación Intercultural, es el proceso cultural y social por el cual se deconstruye-reconstruye la escuela a partir de la heterogeneidad y de las diversas distinciones culturales de todos/as los/las estudiantes, que se resisten a la homogeneización. Para desarrollar esta etapa de valorización de la Alteridad, ambas instituciones deben hacer un esfuerzo en redefinir su Proyecto Educativo Institucional que se fundamente en el cambio en la alternancia recíproca de las culturas que reconoce al distinto como un interlocutor válido, en un proyecto dialógico, ético y político en su Visión y Misión como instituciones educativas.

Una quinta conclusión es el reduccionismo conceptual de la alteridad que poseen los docentes en ambas instituciones, reconocen en una relación intersubjetiva, mediatizada por la buena integración del estudiante inmigrante a lo normativo de la escuela. Pero, a nivel de aula, existe un desarrollo de experiencias curriculares incipientes en valorizar la alteridad del estudiante inmigrante para transmitir sus saberes cognitivos y culturales a su par chileno.

Una sexta conclusión es que en ambas instituciones educativas existe una visión parcializada el sentido comprensivo de Identidad Cultural, prevalece la materialidad cultural del inmigrante, que se instala en los eventos sociales organizados. De esta manera, se reconocen sus bailes, vestimentas, festivos, comidas y modismos propios de la lengua original de los países de los cuales provienen.

Aún falta integrar las otras variables que constituyen la identidad según Bonfil, tales como: las relaciones socioculturales que traen en su convivencia familiar y comunitaria, sus categorías simbólicas que tienen que ver con la religiosidad, creencias, sentimientos de autoafirmación cultural y socio familiar; en fin, las variables que representan los potenciales cognitivos, valóricos y disposiciones para la acción que poseen estos/as estudiantes. Al no conocer estas variables más estructurales y profundas de identidad cultural, reducen el concepto de identidad cultural a las manifestaciones superficiales y folclóricas.

Una séptima conclusión es que en ambas escuelas existe un desarrollo unilateral que privilegia la identidad cultural peruana, se entienden tales manifestaciones folclóricas, socialmente reconocidas desdeñando el aporte diferencial a lo peruano, que pueden hacer las diversas identidades culturales. Este folklorismo pedagógico se encuentra acotado a eventos masivos durante el año escolar, lo cual hace reproducir un enfoque "asimilacionista" en la cotidianidad de la cultura oficial, de tal forma que el estudiante inmigrante logre, en forma rápida, adoptar y adaptarse a la cultura que lo acoge a través de una asimilación forzada en general.

La superficialidad conceptual, en ambas comunidades educativas sobre la Identidad Cultural del estudiante inmigrante, solo confirma la política de integración autorreferente que se aplica en la educación nacional actual. Esta forma de entender la inclusión e integración escolar implica una sumisión y una identificación completa al sistema de valores de la cultura que acoge a aquellos/as estudiantes inmigrantes que se integran.

Una octava conclusión está referida es que la Identidad Cultural que, en su construcción implica, por un lado, la afirmación del sujeto en su pertenencia al grupo que lo identifica; y por otro, a la relación con los otros grupos distintos de su cultura. Es decir, sujetos de diferentes culturas construidas en oposición a su “Otredad". Por lo anteriormente señalado, 
en ambas instituciones educativas se produce un proceso de simetría cultural chilenoperuana y de asimetría cultural con las otras identidades culturales minoritarias.

Para finalizar, ambas escuelas argumentan en forma retórica aceptar la Diversidad Cultural de sus estudiantes, (Multiculturalismo), cuyos principios de igualdad y diferencia están implícitos en sus proyectos educativos, por lo tanto, ambas instituciones educativas, están obligada a realizar un esfuerzo mayor para dar inicio una nueva etapa de consolidación de una verdadera Escuela Intercultural, que conceda al reconocimiento a todas las culturas de sus estudiantes.

\section{REFERENCIAS BIBLIOGRAFICAS}

Cabaluz, F. (2015). Entramando. Editorial Quimantú.

Colombres, A. (1984). (comp.). Una aproximación al problema del control Cultural lo propio y lo ajeno. Premiá Editora.

Dussel, E. (1974). Método para una filosofía de la liberación. Ediciones Sígueme. (1980). La pedagógica latinoamericana. Editorial Nueva América. (1995). Introducción a la filosofía de la liberación. Editorial Nueva América.

Fernández, R. (2012). Una mirada a los derechos de los niños, niñas y adolescentes inmigrantes en Chile. Avances y desafíos pendientes. Ediciones Andros.

Garduño, Everardo. (2010). La Conquista de América: El problema del otro. Culturales, URL. https://www.redalyc.org/pdf/694/69415135008.pdf

Ríos, T. (2013). La Hermenéutica Reflexiva en la investigación educacional y social. Colombia. Universidad de Manizales. Facultad de Ciencias Sociales y Humanas.

Rodríguez, G., Flores, J. G. \& Jiménez, E. G. (1996). Metodología de la investigación cualitativa. Ed. Aljibe. Málaga.

Santander, P. (2011). Por qué y cómo hacer Análisis de Discurso. URL. www.moebio.uchile.cl/41/ santander.html

Todorov, Tzvetan. (2003). La Conquista de América. El problema del otro (trad. Flora Botton). Editores Siglo XXI. 
